\title{
Antimicrobial Resistance Trends in Staphylococcus aureus Strains Carried by Poultry in North of Morocco: A Preliminary Analysis
}

\author{
Nadira Mourabit $\mathbb{D}^{1},{ }^{1}$ Abdelhay Arakrak, ${ }^{2}$ Mohammed Bakkali, ${ }^{2}$ Zeineb Zian, ${ }^{3}$ \\ Joaira Bakkach, ${ }^{3}$ and Amin Laglaoui ${ }^{2}$ \\ ${ }^{1}$ Higher Institute of Nursing Professions and Technical Health of Tangier, Tetouan, Morocco \\ ${ }^{2}$ Biotechnology and Biomolecule Engineering Research Laboratory, Faculty of Sciences and Techniques of Tangier, \\ Abdelmalek Essaadi University, Tetouan, Morocco \\ ${ }^{3}$ Biomedical Genomics and Oncogenetics Research Laboratory, Faculty of Sciences and Techniques of Tangier, \\ AbdelmalekEssaadi University, Tetouan, Morocco \\ Correspondence should be addressed to Nadira Mourabit; nadirauniver@gmail.com
}

Received 15 June 2020; Revised 10 December 2020; Accepted 15 May 2021; Published 24 May 2021

Academic Editor: Jordi Rovira

Copyright (C) 2021 Nadira Mourabit et al. This is an open access article distributed under the Creative Commons Attribution License, which permits unrestricted use, distribution, and reproduction in any medium, provided the original work is properly cited.

\begin{abstract}
The transmission of antibiotic resistance to human population through food consumption is a global public health threat. This study aimed to assess the nasopharyngeal carriage of $S$. aureus in poultry and to investigate antimicrobial susceptibility and virulence-associated genes. Nasopharyngeal swabs were collected from chickens at the slaughterhouse of Tangier and immediately transported to the microbiological laboratory for phenotypic identification and assessment of antibiotic susceptibility. The presence of 16S rRNA, nuc, mecA, mecC, Panton-Valentine leukocidin (PVL), and the toxic shock syndrome toxin 1 (TSST-1) genes were detected by PCR analysis for all isolates. Overall, 548 nasopharyngeal swabs were collected, of which 17 (3.4\%) were S. aureus positive. More than half of the strains (54\%) were resistant to penicillin, $29.4 \%$ to tetracycline, $23.5 \%$ to erythromycin, and $17 \%$ showed resistance to ciprofloxacin. The mecA and mecC were not identified in any of the recovered isolates. Of the S. aureus recovered, $29.41 \%$ of the isolates were found to be toxinogenic; $17.64 \%$ and $11.76 \%$ were positive for PVL and TSST-1 encoding genes, respectively. The trends of antibiotic resistance and the toxinogenic $S$. aureus carried by the poultry intended for consumption in Tangier present a huge concern. Preventive and containment measures should be implemented in order to limit the dissemination of resistance genes through the food chain and to reduce their increased rate.
\end{abstract}

\section{Introduction}

As stated by the World Health Organization (WHO), antibiotic resistance (AR) is considered as a rising global human health threat $[1,2]$. According to the Centers for Disease Control and Prevention (CDC), at least 2.8 million individuals get an antibiotic-resistant infection, and more than 35,000 individuals died as a result [3]. A British study on antimicrobial resistance (AMR) estimated that 700,000 persons are dying each year worldwide due to antibiotic-resistant infections [4].

Industrial food animal production facilities are known to be a source of human exposure to antibiotic-resistant
S. aureus including methicillin-resistant $S$. aureus (MRSA) $[5,6]$. This system uses intensive confinement that requires a regular implementation of antibiotics for therapeutic and/or preventive purposes, in tandem with using antibiotics as growth promoters in poultry production systems in order to ensure sufficient amount of food to an ever-growing world population $[5,7,8]$. The enormous use of animals intended for food promotes the selection of new antibiotics resistant bacteria, which will contribute to increasing the risk of zoonotic transmission [6]. Zoonotic resistant bacteria and resistance genes could be transferred not only to people with occupational livestock exposure but also other persons in the 
community through direct contact with animals, via the food chain or by environment $[9,10]$.

$S$. aureus is a pathogen, which is known to colonize and infect both humans and animals and to contaminate food as well $[6,11,12]$. The pathogenicity of $S$. aureus is related to the expression of a large number of virulence factors that promote adhesion and evasion of the host immunologic responses [13]. Certain toxins are strongly associated with specific diseases and a high mortality rate, such as toxic shock syndrome toxin (TSST) and Panton-Valentine leukocidin (PVL) toxin, which is directly involved in the pathology of necrotizing pneumonia and inducing leukocytosis and tissue necrosis [12-14].

The evolution of $S$. aureus in the antibiotic era has revealed the emergence of virulent strains, many of which include acquisition of antibiotic resistant to methicillin $[15,16]$. The high burden of MRSA has been first reported in healthcare facilities, second in community, and last in livestock settings worldwide [6, 10, 15-18].

MRSA strains may spread in all geographic regions between different ecological niches resulting in major healthcare costs $[2-4,6,9,10,17]$ and relevant economic losses in the food animal industry [2, 11, 15-17].

Despite the significant potential consequences for AMR in livestock, our knowledge about antibiotic-resistant S. aureus carriage among food-producing animals in Tangier is still limited.

To the best of our knowledge, the present study aimed to determine, for the first time in Tangier, S. aureus nasopharyngeal carriage in poultry to describe antimicrobial susceptibility and the distribution of TSST and PVL toxins genes among the recovered strains.

\section{Materials and Methods}

2.1. Isolation of $S$. aureus and MRSA from Chickens. Sampling was performed from February to May 2016 from large chicken slaughterhouse in Tangier. Nasopharyngeal samples were randomly taken in batches from chickens using common sterile dry cotton swabs (Euroturbo, Deltalab, Spain) immediately after slaughter. All samples were stored at $4^{\circ} \mathrm{C}$ until be analyzed by phenotypic and molecular tests.

2.2. Detection of S. aureus and MRSA. Swabs were inoculated into $10 \mathrm{ml}$ of Mueller-Hinton broth (Oxoid, Basingstoke, $\mathrm{UK}$ ) containing $6.5 \% \mathrm{NaCl}$, then incubated overnight at $37^{\circ} \mathrm{C}$, and subcultured on selective media chromogen (UriSelect $^{\mathrm{TM}} 4$ Medium). One $S$. aureus colony was streaked for isolation per a plate.

Phenotypic identification was further performed for presumptive $S$. aureus colonies by catalase, Gram staining, and DNase test.

2.3. Molecular Identification by PCR. All isolates were screened by PCR analysis for the presence of the genes coding 16S rRNA, nuc, and mecA [19]. Resistance to methicillin was also tested for the presence of the mec $\mathrm{C}$ genes by PCR assays as previously described by García-Álvarez et al. [20].

Screening for the presence of the genes coding for PVL and TSST- 1 was carried out using PCR as reported by Denis et al. [21]. S. aureus strains ATCC 29213 (nuc-positive), ATCC 2011S359 (mecC-positive), ATCC 43300 (mecApositive), FRI913 (tst-positive), and MW2 (lukS/F-PVpositive) were used as positive controls for PCR.

2.4. Antibiotic Susceptibility Testing. All the S. aureus isolates obtained were assessed for susceptibility with 11 antibiotics: penicillin, cefoxitin, tetracycline, chloramphenicol, sulfamethoxazole/trimethoprim, clindamycin, erythromycin, ciprofloxacin, fusidic acid, gentamicin, and kanamycin. Breakpoints established for each antimicrobial susceptibility were used according to the European Committee on Antimicrobial Susceptibility Testing (EUCAST) recommendations [22]. Susceptibility for vancomycin (vancomycin hydrochloride; Sigma-Aldrich; Lot\#050M1312 V) MICs was determined by broth microdilution for all isolates using the method adapted from EUCAST [22].

\section{Results}

3.1. Antibiotic Susceptibility. S. aureus was detected in 17 out of the 542 tested samples (3.1\%). We found that approximately two-thirds of the isolates (11/17, 64.7\%) exhibited resistance to one antibiotic, and eight isolates (47.05\%) showed resistance to at least two antibiotics. Multidrug resistance patterns of the methicillin-susceptible $S$. aureus strains are presented in Table 1. Overall, nine isolates $(52.94 \%)$ were resistant to penicillin, five isolates (29.4\%) to tetracycline, four $(23.5 \%)$ to erythromycin, and three isolates showed resistance (17.64\%) to ciprofloxacin. None of the $17 \mathrm{~S}$. aureus isolates were resistant to cefoxitin, clindamycin, chloramphenicol, gentamicin, kanamycin, sulfamethoxazole/trimethoprim, and vancomycin.

3.2. Toxin Genes Screening. Overall, from the 17 S. aureus recovered, five $(29.41 \%)$ isolates were toxinogenic, three of which $(17.64 \%)$ showed positive results for PVL, and two (11.76\%) for TSST-1 encoding genes. None of these S. aureus colonies carried out mecA and mecC genes.

\section{Discussion}

Several studies with particular focus on the risk of foodborne transmission of antimicrobial resistance have reported the presence of $S$. aureus in poultry with different rates $[18,23-26]$.

The present study demonstrated a low prevalence of S. aureus nasopharyngeal carriage $(17 / 542 ; 3.1 \%)$ in chickens. Unfortunately, we could not compare our findings to Moroccan data as no studies on nasopharyngeal carriage of $S$. aureus in poultry are available in the national scale. We recently published a study [27] carried out in Tangier in order to identify the nasal carriage rate of $S$. aureus in farm animals. However, comparing both studies is quite difficult. 
TABLE 1: Multidrug resistant pattern of the Staphylococcus aureus strains carried by poultry.

\begin{tabular}{lc}
\hline Resistance pattern & No. of resistant isolates $\left(n^{*}(\%)\right)$ \\
\hline P & $02(11.76)$ \\
E & $01(5.88)$ \\
E, P & $03(17.64)$ \\
Te, P & $02(11.76)$ \\
Cip, P & $01(5.88)$ \\
E, Te, P & $01(5.88)$ \\
Cip, Te, P & $11(64.70)$ \\
Total & $01(5.88)$ \\
\hline
\end{tabular}

Cip, ciprofloxacin; E, erythromycin; P, penicillin; Te, tetracycline. ${ }^{*}$ The number of isolates tested with the percentage of resistance in parenthesis (\%).

In our previous work, animal farms did not have a history of antibiotic therapy during three months prior to the screening; in contrast, we do not have information about using antibiotic therapy in this work.

Our prevalence of $S$. aureus nasopharyngeal carriage in poultry remains very lower compared to a study conducted in different Algerian areas that reported a rate which varied by poultry species and ranged between $48.4 \%$ and $73.6 \%$ [23]. Another study from Nigeria described a rate of $38.33 \%$ in chickens [25]. Higher rates of $S$. aureus varying between $40 \%$ and $54 \%$ were found in broiler chicken slaughterhouses and retail outlets around the Durban metropolitan area in South Africa [24].

Data of occurrence and susceptibility of strains have been shown to change over time, depending on the country of origin, resources settings, poultry specimens, methods of sampling, and hygienic practices during slaughtering $[23,24,26]$. A meta-analysis aiming to determine the overall occurrence of $S$. aureus in the poultry production in various geographic areas [26], revealed that pooled MRSA prevalence was $16 \%$ in Africa, $15 \%$ in Europe, $27 \%$ in South America, $1 \%$ in North America, and 2\% in Asia. Based on molecular typing, data from this meta-analysis suggested that MRSA strains may spread along the production chain from animals to humans or vice versa. A higher frequency of MRSA isolated from poultry and poultry products has been described in several African countries, which is not in agreement with our results, Algeria with 36\% [23], Nigeria with 38.33\% [25], and South Africa with 32.60\% [24]. Whether the presence of these opportunistic pathogens on poultry meat was before/immediately after slaughter or as a consequence of possible contamination by meat handlers, it remains a threat to food safety and a great concern for public health [5]. No MRSA strains were found in our study.

In our study, we showed that $52.94 \%$ was resistant to penicillin, $29.4 \%$ to tetracycline, $23.5 \%$ to erythromycin, and $17.64 \%$ of isolates were resistant to ciprofloxacin. Whereas, none of our isolates was resistant to cefoxitin, clindamycin, chloramphenicol, gentamicin, kanamycin, sulfamethoxazole/trimethoprim, and vancomycin. Our drug resistance patterns are generally lower than published data. Strains recovered from poultry in Mkhize et al. study exhibited higher rate of resistance to tetracycline (79.4\%), followed by ampicillin (65.1\%), cefoxitin $60.3 \%$, and erythromycin $57.1 \%$
[24]. Another recent report by Benrabia et al. showed resistance to tetracycline in $82.5 \%$, erythromycin in $70.6 \%$, clindamycin in $68.6 \%$, and ciprofloxacin in $50 \%$ of cases [23]. However, the majority of our drug resistance patterns remains higher than findings from another study carried out on oral carriage poultry by Mairi et al., who reported antimicrobial resistance rates of $73.9 \%, 4.3 \%, 1.4 \%, 8.6 \%$, and $1.4 \%$ observed for penicillin, cefoxitin, ofloxacin, erythromycin, and minocycline, respectively [18]. The rates of antimicrobial resistance in the current study were higher than those we previously reported in farm animals $(29.4 \%$ and $23.5 \%$ versus $16.7 \%$ and $11.9 \%$ for tetracycline and macrolides, respectively). Interestingly, we observed a resistance rate of $17.64 \%$ for ciprofloxacin in the present work, while this antimicrobial resistance was not present in the aforementioned study [27].

In general, strains carried by poultry intended for consumption in Tangier showed lower resistance rates compared to those listed above in the majority of African countries [23-25]. However, the presented rates cause huge concern given the nature of the molecules affected by resistance and the potential consequences that can result if preventive and containment measures are not taken to limit the increase rate and dissemination of resistance genes. In fact, these resistance genes can be exchanged between bacteria of the same host by horizontal transfer [28], as well as they can escape the barriers to reach other species by direct contact with animals or through the food chain to the community and to the environment $[6,10]$.

Interestingly, it seems that the antibiotics showing resistance are those used in poultry farming worldwide and in Morocco [7, 29]. The frequent use of this type of antibiotics and constant exposure can create a selective environment for mutants resistant to these antibiotics. Therefore, these resistances can translate a massive use in our institution or can be used as a marker to follow the zoonotic transmission $[30,31]$. In addition, the resistance observed among poultry isolates to fluoroquinolones confirms that the big problem in Morocco is the inappropriate use of this molecule in human medicine, veterinary medicine, or inbreeding which leads to developing a resistance to various bacteria such as $S$. aureus and other strains of the intestinal microbiota $[7,8,29,32]$.

Aware of the burden of antibiotic resistance, Morocco has tried to regulate poultry farming since 2002 by law (49-99; 2002). The National Office for Health Security of Food Products (ONSSA) through its food safety monitoring program confirmed that poultry farming fulfills international safety standards [33]. However, the surveillance and control system remains insufficient and still facing several challenges, particularly in rationalizing the antibiotic use and containing the prescription of antibiotics for a curative, preventive, or fattening purpose $[7,8]$. The overuse of antimicrobial agents, especially tetracycline, in food animal production such as growth stimulating antibiotics, is considered as one of the most important practices, which may induce selection of resistant mutants [7, 8]. It is still possible for Morocco to contain easily the problem of antibiotic resistance. Still, we should seriously consider implementing the "One Health" concept which recommended effective 
multisectoral, multidisciplinary, and transnational collaboration in all aspects of healthcare for humans, animals, and the environment $[34,35]$.

Beyond resistance, the relatively higher rate of toxinogenic $S$. aureus strains recovered from chickens gained a great interest from many researchers due to their impact in animal and human health $[10,14,18]$. The occurrence of the same virulence genes carried by healthy humans in the community [36] or by farm animals in the same geographical area [27] showed the possibility of dissemination and distribution of such virulence genes [10, 12]. Although the phylogenetic relationship between the different strains has not been evaluated, the ability of virulence genes to cross the species barrier might be suggested.

Our work presented some limitations. It is a monocentric study, which was carried out for a short duration and identified a lower number of isolates. Thus, it is difficult to consider this study as epidemiology relevant. The absence of molecular typing technologies for the identification of the epidemic clones of this pathogen is another limitation of our work. Hence, we could not provide firm conclusions about the nasopharyngeal carriage of $S$. aureus, antibiotic resistance patterns, and the spread of virulence genes in poultry in our geographic area.

\section{Conclusion}

In conclusion, our study presented, to the best of our knowledge, for the first time in north of Morocco, the patterns of antibiotic resistance and revealed the presence of a higher rate of toxinogenic $S$. aureus in poultry intended for human consumption. These findings require further investigation. Assessing the risk of transmission of antibiotic resistance through the food chain is of great importance in order to manage this global burden.

\section{Data Availability}

The data used to support the findings of this study are available from the corresponding author upon request.

\section{Conflicts of Interest}

The authors declare that they have no conflicts of interest.

\section{Acknowledgments}

The authors would like to acknowledge the poultry slaughterhouse, which allowed us to collect samples for this research. The authors would also like to acknowledge all the staff for assisting them during sampling.

\section{References}

[1] World Health Organization, "Antibiotic resistance. Geneva, Switzerland," World Health Organization, Geneva, Switzerland, 2019.

[2] M. Ahmad and A. U. Khan, "Global economic impact of antibiotic resistance: a review," Journal of Global Antimicrobial Resistance, vol. 19, pp. 313-316, 2019.
[3] J. O'Neill, Antimicrobial Resistance: Tackling a Crisis for the Health and Wealth of Nations, World Health Organization, Geneva, Switzerland, 2014.

[4] Centers for Disease Control and Prevention (CDC) (U.S), Antibiotic Resistance Threats in the United States, Centers for Disease Control and Prevention (CDC) (U.S), Atlanta, GA, USA, 2019.

[5] E. K. Silbergeld, J. Graham, and L. B. Price, "Industrial food animal production, antimicrobial resistance, and human health," Annual Review of Public Health, vol. 29, no. 1, pp. 151-169, 2008.

[6] M. Nemati, K. Hermans, U. Lipinska et al., "Antimicrobial resistance of old and recent Staphylococcus aureus isolates from poultry: first detection of livestock-associated methicillin-resistant strain ST398', Antimicrobial Agents Chemotherapy, vol. 52, pp. 103817-103819, 2008.

[7] N. Rahmatallah, H. El Rhaffouli, I. Lahlou Amine, Y. Sekhsokh, O. Fassi Fihri, and M. El Houadfi, "Consumption of antibacterial molecules in broiler production in Morocco," Veterinary Medicine and Science, vol. 4, no. 2, pp. 80-90, 2018.

[8] K. Es-Soucratti, A. Hammoumi, B. Bouchrif, R. Asmai, H. EnNassiri, and B. Karraouan, "Occurrence and antimicrobial resistance of Campylobacter jejuni isolates from poultry in Casablanca-Settat, Morocco," Italian Journal of Food Safety, vol. 9, no. 1, p. 8692, 2020.

[9] L. E. Spoor, P. R. McAdam, L. A. Weinert et al., "Livestock origin for a human pandemic clone of community-associated methicillin-resistant Staphylococcus aureus," MBio, vol. 4, no. 4, pp. e00356-13, 2013.

[10] S. Wu, F. Zhang, J. Huang et al., "Phenotypic and genotypic characterization of PVL-positive Staphylococcus aureus isolated from retail foods in China," International Journal of Food Microbiology, vol. 304, pp. 119-126, 2019.

[11] J. M. Fueyo, M. C. Mendoza, M. R. Rodicio, J. Muniz, M. A. Alvarez, and M. C. Martin, "Cytotoxin and pyrogenic toxin superantigen gene profiles of Staphylococcus aureus associated with subclinical mastitis in dairy cows and relationships with macrorestriction genomic profiles," Journal of Clinical Microbiology, vol. 43, no. 3, pp. 1278-1284, 2005.

[12] M. Vitale, M. L. Scatassa, C. Cardamone et al., "Staphylococcal food poisoning case and molecular analysis of toxin genes inStaphylococcus aureusStrains isolated from food in sicily, Italy," Foodborne Pathogens and Disease, vol. 12, no. 1, pp. 21-23, 2015.

[13] D. Grumann, U. Nübel, and B. M. Bröker, "Staphylococcus aureus toxins - their functions and genetics," Infection, Genetics and Evolution, vol. 21, pp. 583-592, 2014.

[14] L. J. Shallcross, E. Fragaszy, A. M. Johnson, and A. C. Hayward, "The role of the Panton-Valentine leucocidin toxin in staphylococcal disease: a systematic review and metaanalysis," The Lancet Infectious Diseases, vol. 13, no. 1, pp. 43-54, 2013.

[15] M. Gajdács, "The continuing threat of methicillin-resistant Staphylococcus aureus," Antibiotics, vol. 8, no. 2, p. 52, 2019.

[16] C. C. Dweba, O. Zishiri, and M. El Zowalaty, "Methicillinresistant Staphylococcus aureus: livestock-associated, antimicrobial, and heavy metal resistance," Infection and Drug Resistance, vol. 11, pp. 2497-2509, 2018.

[17] M. Aires-de-Sousa, "Methicillin-resistant Staphylococcus aureus among animals: current overview," Clinical Microbiology and Infection, vol. 23, no. 6, pp. 373-380, 2017.

[18] A. Mairi, A. Touati, A. Pantel et al., "Distribution de Staphylococcus aureus toxique et résistant à la méthicilline et 
sensible à la méthicilline dans différents niches écologiques en Algérie," Toxines (Bâle), vol. 11, no. 9, p. 500, 2019.

[19] N. Maes, J. Magdalena, S. Rottiers, Y. De Gheldre, and M. J. Struelens, "Evaluation of a triplex PCR assay to discriminate Staphylococcus aureus from coagulase-negative staphylococci and determine methicillin resistance from blood cultures," Journal of Clinical Microbiology, vol. 40, no. 4, pp. 1514-1517, 2002.

[20] L. García-Álvarez, M. T. Holden, H. Lindsay et al., "Meticillinresistant Staphylococcus aureus with a novel mecA homologue in human and bovine populations in the UK and Denmark: a descriptive study," The Lancet Infectious Diseases, vol. 11, no. 8, pp. 595-603, 2011.

[21] O. Denis, A. Deplano, H. De Beenhouwer et al., "Polyclonal emergence and importation of community-acquired methicillin-resistant Staphylococcus aureus strains harbouring Panton-Valentine leucocidin genes in Belgium," Journal of Antimicrobial Chemotherapy, vol. 56, no. 6, pp. 1103-1106, 2005.

[22] The European Committee on Antimicrobial Susceptibility Testing, Breakpoint Tables for Interpretation of MICs and Zone Diameters, The European Committee on Antimicrobial Susceptibility Testing, Växjö, Sweden, 2015, https://www. eucast.org/fileadmin/src/media/PDFs/EUCAST_files/ Breakpoint_tables/v_5.0_Breakpoint_Table_01.pdf.

[23] I. Benrabia, T. M. Hamdi, A. A. Shehata, H. Neubauer, and G. Wareth, "Methicillin-resistant Staphylococcus aureus (MRSA) in poultry species in Algeria: long-term study on prevalence and antimicrobial resistance," Vet Science, vol. 7, no. 2, p. E54, 2020.

[24] N. Mkhize, O. T. Zishiri, and S. Mukaratirwa, "Genetic characterisation of antimicrobial resistance and virulence genes in Staphylococcus aureus isolated from commercial broiler chickens in the Durban metropolitan area, South Africa," Journal of the South African Veterinary Association, vol. 88, no. 1, pp. 1-7, 2017.

[25] I. Kwoji, S. Jauro, J. Musa, Y. Lekko, S. Salihu, and H. Danchuwa, "Phenotypic detection of methicillin-resistant Staphylococcus aureus in village chickens from poultry markets in Maiduguri, Nigeria," Journal of Advanced Veterinary and Animal Research, vol. 6, no. 2, p. 163, 2019.

[26] C. M. Ribeiro, L. M. Stefani, S. B. Lucheis et al., "Methicillinresistant Staphylococcus aureus in poultry and poultry meat: a meta-analysis," Journal of Food Protection, vol. 81, no. 7, pp. 1055-1062, 2018.

[27] N. Mourabit, A. Arakrak, M. Bakkali, Z. Zian, J. Bakkach, and A. Laglaoui, "Nasal carriage of Staphylococcus aureus in farm animals and breeders in North of Morocco," BMC Infect Dis, vol. 20 , no. 1 , p. $602,2020$.

[28] F. C. Tenover, "Mechanisms of antimicrobial resistance in bacteria," American Journal of Infection Control, vol. 34, no. 5, Suppl1, 2006.

[29] World Organization for Animal Health (OIE), List of Antimicrobial Agents of Veterinary Importance, World Organization for Animal Health, Geneva, Switzerland, 2015.

[30] V. Bortolaia and L. Guardabassi, "Zoonotic transmission of antimicrobial resistant enterococci: a threat to public health or an overemphasised risk?," in Zoonoses-Infections Affecting Humans and Animals Zoonoses: Infections Affecting Men and Animals-a Focus on Public Health Aspects, A. Sing, Ed., Springer Netherlands, Berlin, Germany, pp. 407-431, 2015.

[31] I. Montesinos, E. Salido, T. Delgado, M. Cuervo, and A. Sierra, "Epidemiologic genotyping of methicillin-resistant Staphylococcus aureus by pulsed-field gel electrophoresis at a university hospital and comparison with antibiotyping and protein A and coagulase gene polymorphisms," Journal of Clinical Microbiology, vol. 40, no. 6, pp. 2119-2125, 2002.

[32] T. Berradi et al., "Résistance aux fluoroquinolones des Salmonella Kentucky isolées de produits alimentaires d'origine animale (Casablanca, Maroc)," Medicine Maladies Infection, 2018.

[33] Office National de Sécurité Sanitaire des Aliments (ONSSA), Requirements for Poultry Production, Office national de sécurité sanitaire des aliments (ONSSA), 2015, http://www. onssa.gov.ma/fr/index.php?option $=$ com content\&view $=$ article\&id $=187 \&$ Itemid $=126$.

[34] R. Laxminarayan, A. Duse, C. Wattal et al., "Antibiotic resistance-the need for global solutions," The Lancet Infectious Diseases, vol. 13, no. 12, pp. 1057-1098, 2013.

[35] One Health, 2019, https://www.cdc.gov/onehealth/index. html.

[36] N. Mourabit, A. Arakrak, M. Bakkali, and A. Laglaoui, "Nasal carriage of sequence type 22 MRSA and livestock-associated ST398 clones in Tangier, Morocco," The Journal of Infection in Developing Countries, vol. 11, no. 7, pp. 536-542, 2017. 